\title{
Potential Vorticity Diagnosis of the Severe Convective Regime. Part II: The Impact of Idealized PV Anomalies
}

\author{
John W. Nielsen-Gammon And David A. Gold* \\ Department of Atmospheric Sciences, Texas A\&M University, College Station, Texas
}

(Manuscript received 16 November 2006, in final form 17 September 2007)

\begin{abstract}
Idealized numerical experiments are conducted to understand the effect of upper-tropospheric potential vorticity (PV) anomalies on an environment conducive to severe weather. Anomalies are specified as a single isolated vortex, a string of vortices analogous to a negatively tilted trough, and a pair of string vortices analogous to a position error in a negatively tilted trough. The anomalies are placed adjacent to the tropopause along a strong upper-level jet at a time just prior to a major tornado outbreak and inverted using the nonlinear balance equations.

In addition to the expected destabilization beneath and adjacent to a cyclonic PV anomaly, the spatial pattern of the inverted balanced streamfunction and height fields is distorted by the presence of the horizontal PV gradient along the upper-tropospheric jet stream. Streamfunction anomalies are elongated in the cross-jet direction, while height and temperature anomalies are elongated in the along-jet direction. The amplitude of the inverted fields, as well as the changes in CAPE associated with the inverted temperature perturbations, are linearly proportional to the amplitudes of the PV anomalies themselves, and the responses to complex PV perturbation structures are approximated by the sum of the responses to individual simple PV anomalies. This is true for the range of PV amplitudes tested, which was designed to mimic typical 6-h forecast or analysis errors and produced changes in CAPE beneath the trough of well over $100 \mathrm{~J} \mathrm{~kg}^{-1}$. Impacts on inverted fields are largest when the PV anomaly is on the anticyclonic shear side of the jet, where background PV is small, compared with the cyclonic shear side of the jet, where background $\mathrm{PV}$ is large.
\end{abstract}

\section{Introduction}

The approach of an upper-level trough in the central United States in spring or summer may be expected to increase the probability of severe weather there. A trough is typically accompanied by cooling aloft, and the large-scale ascent ahead of the trough may be expected to increase the convective available potential energy (CAPE) and decrease the convective inhibition (CIN). The deep southerly winds ahead of the trough may be expected to enhance the transport of moisturerich low-level air into the area, further enhancing CAPE. Finally, the enhanced vertical wind shear ac-

* Current affiliation: PPM Energy, Inc., Houston, Texas.

Corresponding author address: John W. Nielsen-Gammon, Department of Atmospheric Sciences, Texas A\&M University, 3150 TAMU, College Station, TX 77843-3150.

E-mail: n-g@tamu.edu companying the tropospheric temperature gradients associated with the trough may be expected to enhance the vertical wind shear that favors multicellular and supercellular thunderstorms.

If these effects are proportional to trough intensity, as they may appear to be, a stronger trough would be expected to lead to a greater likelihood of severe weather. However, there is considerable variability in severe weather from event to event, much more than the apparent variability in upper-tropospheric flow structure, suggesting that either the upper-tropospheric systems do not exert a controlling influence, or alternatively, that the severe weather is extremely sensitive to details of the upper-tropospheric weather pattern.

Diagnostic and modeling studies are useful for addressing these issues in the context of specific cases. Such case studies must be combined with a general basic knowledge of the effect of simple variations of upper-tropospheric structure on convective morphology and intensity, in order that the knowledge gained from such studies may be understood and applied to the 
spectrum of future events that arises in a forecasting environment.

This paper is Part II of a four-part series that uses potential vorticity (PV) inversion to diagnose the sensitivity of the severe convective regime (SCR) to changes in the upper-tropospheric PV field. Gold and Nielsen-Gammon 2008a (hereafter Part I) described the inversion and sounding reconstruction methodology, and Gold and Nielsen-Gammon 2008b,c (hereafter Parts III, IV) contain case studies of two major severe weather outbreaks. The purpose of this paper is to develop the general basic knowledge of the effect of structural PV changes on the convective environment. This is accomplished by inverting idealized PV structures embedded within an environment supporting severe weather, and examining the resulting modifications to the atmosphere, including the effects on the CAPE distribution.

The present paper is relevant in a more general context as well, as the first to investigate the atmospheric response to idealized PV perturbations in a horizontally inhomogeneous environment. During a severe weather episode, one or more upper-tropospheric mobile troughs are typically embedded in a strong upper-level jet stream characterized by a nearly vertical tropopause and strong horizontal gradients of potential vorticity (Shapiro and Keyser 1990). The presence of these strong horizontal PV gradients will be shown to lead to substantial changes in the structure and intensity of the balanced atmospheric response to a PV anomaly. Here and elsewhere, "balanced" refers to that portion of the time-dependent flow recoverable from PV inversion, following Davis et al. (1996).

Thorpe (1986) produced well-known diagrams of the balanced atmospheric structure associated with a circular vortex on the tropopause. Juckes (1999) developed analytical solutions for two-dimensional (linear) tropopause-based anomalies, which are idealized representations of elongated troughs or PV filaments. Juckes and Smith (2000), motivated by the interaction between transitioning hurricanes and extratropical uppertropospheric troughs, looked specifically at the CAPE and CIN directly beneath such troughs compared with their environments, and found destabilization manifested in both parameters as a nonlinear response to trough intensity. In all of these studies, the PV anomalies were embedded in an environment with no horizontal variations.

Previous studies have also examined the effect of actual PV anomalies within model simulations of specific events. Griffiths et al. (2000) studied the potential destabilizing effect of a tropopause fold by analyzing the effect of the fold on advection of wet-bulb potential temperature. Roebber et al. (2002) examined the effect of an upper-tropospheric mobile trough by using PV inversion to alter it in the initial condition of the model and examining the subsequent model simulation of convection.

Because we will be dealing with idealized, isolated PV anomalies, we will make use of the theoretical studies of Bishop and Thorpe (1994) and Thorpe and Bishop (1995). These papers used a Green's function approach to develop an electrostatics analogy to PV inversion. They were particularly interested in the effect of vertical variations of the background PV field, but the techniques they developed will be useful here in the context of the effect of horizontal variations of the background PV field on the inverted fields.

This paper is organized as follows: in section 2, a conceptual model is used to describe the fundamental physical relationship between an idealized PV anomaly and the vertical stratification, wind shear, and forced ascent. Some issues associated with the application of idealized PV inversion to diagnosing convective parameters are discussed in section 3 . Section 4 presents a set of idealized experiments in which the effects of nonlinearity and background PV gradients on CAPE are investigated. The results are summarized in section 5 .

\section{A simple conceptual model linking PV to convective parameters}

To illustrate how PV changes affect the vertical distributions of wind and temperature relevant to moist convection, it is helpful to first conceptualize the spatial relationship between these elements and an idealized PV anomaly. A schematic representing a cross section in the $x-z$ plane through an idealized spherical PV anomaly is presented in Fig. 1. This PV anomaly $\delta q$, when added to a horizontally uniform background PV distribution $q_{0}=f_{0} / \rho(d \bar{\theta} / d z)$, results in the familiar distribution of isentropes and winds shown in Fig. 1. This idealized PV "ball" is conceptually similar to the coldcore upper cyclone described in Thorpe (1986, refer to his Fig. 1), featuring isentropes that bend upward (downward) beneath (above) the anomaly, and possessing a core of high static stability $\left(N^{2}\right)$ and vorticity $(\zeta)$. The magnitude of the temperature anomaly (and associated vertical displacement of isentropes from their reference positions) decays vertically and horizontally away from the top and bottom of the PV anomaly, with zero thermal anomalies at the lower and upper boundaries, at the level of the anomaly itself, and at sufficient horizontal distance from the PV anomaly. The isentropic structure shown is associated with anomalously low temperatures and static stability in the 


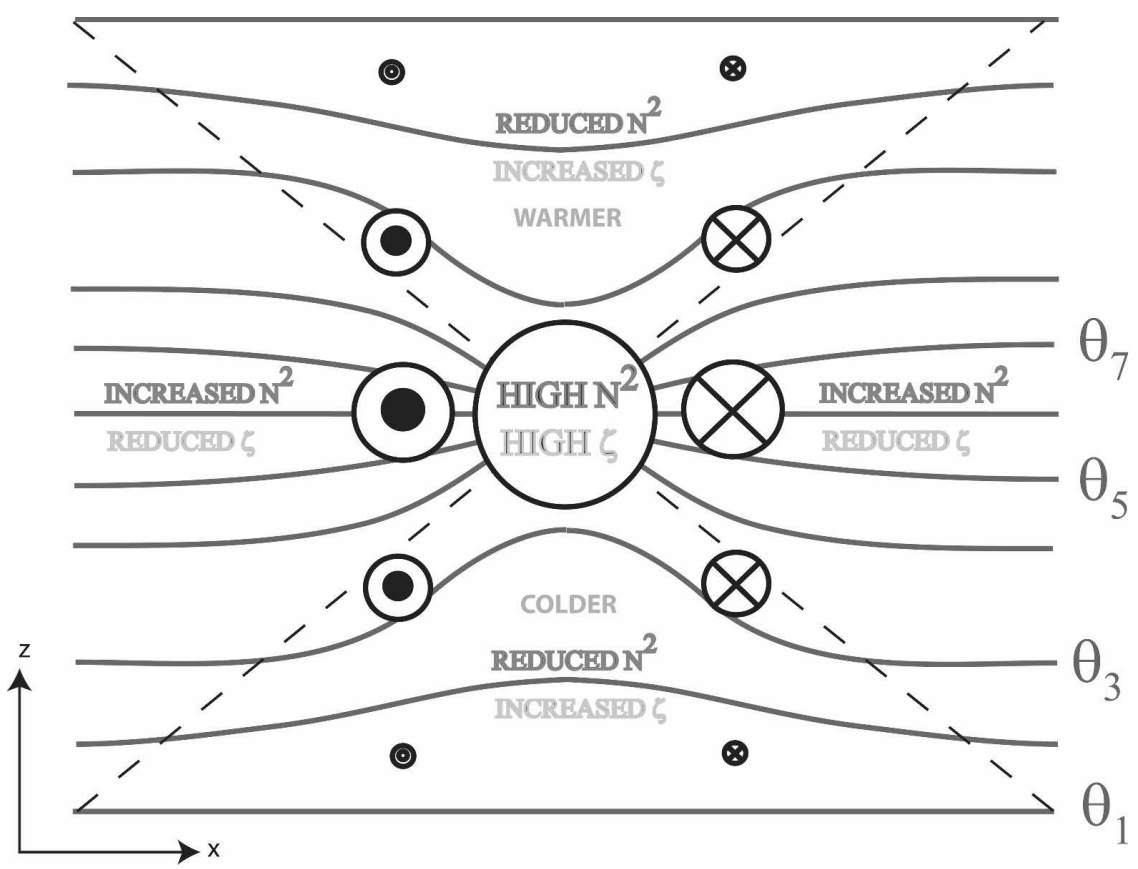

FIG. 1. Idealized schematic of an isolated cyclonic spherical PV anomaly in a uniform background state. The vertical axis is scaled by $N_{0} / f_{0}$.

column beneath the PV anomaly. Cyclonic $\zeta$ is maximized within the layer occupied by the PV anomaly, decaying away above and below that layer.

The thermal structure associated with the idealized PV anomaly depicted in Fig. 1 and the cold-core trough of Thorpe (1986) suggest that the atmosphere beneath the PV anomaly is characterized by increased convective instability. Juckes and Smith (2000) show that cold tropopause thermal anomalies $\theta_{\mathrm{tr}}^{\prime}$ [which correspond to positive perturbation PV on isentropic surfaces (Morgan and Nielsen-Gammon 1998 and references therein)] indeed result in increased CAPE and reduced CIN within the columns immediately beneath and near the PV anomalies. At increasing horizontal distances from the trough axis, these CAPE and CIN perturbations decay.

The distribution of winds attributable to the tropopause anomaly illustrated in Fig. 1 also suggests the possibility that an upper-level trough can give rise to vertical wind profiles conducive to rotating convective updrafts [see Klemp (1987) for a review of the theory relating vertical wind shear to updraft rotation]. Specifically, the perturbation winds $\mathbf{V}^{\prime}$ increase with respect to height within air columns beneath the flanks of the PV anomaly. The implications of these types of wind shear changes on convection depend sensitively on the background wind field and will be discussed in the context of case studies in two companion papers (Parts III and IV).
A change in the amplitude or geometrical configuration of $\delta q$ will result in a corresponding change in the CAPE and/or vertical shear. For example, if the PV anomaly is strengthened, isentropes will be displaced upward to an even greater extent beneath the PV anomaly and the underlying troposphere will cool accordingly. This will result in greater CAPE and reduced CIN, thereby increasing the possibility that deep moist convection will ensue, and that any convection that does develop may be more intense (due to greater CAPE). Likewise, an amplified PV anomaly will be associated with a concomitant increase in the magnitude of $\mathbf{V}^{\prime}$ and its vertical shear, possibly affecting the resulting convective morphology. A change in the horizontal structure of the PV anomaly will change both the spatial distribution and magnitude of the CAPE and shear, changing the strength of these parameters at distances farther from or closer to the trough axis, depending on whether the anomaly becomes horizontally narrower or wider along a given direction. A change in the aspect ratio of the anomaly will alter the relative magnitudes of CAPE and shear impacts, with a tall, narrow PV anomaly associated primarily with wind anomalies and a broad, shallow PV anomaly associated primarily with thermal anomalies (Wirth 2000).

One final effect that impacts convection, and one that is implicit in the structure of the isentropes in Fig. 1, following the arguments of Hoskins et al. (1985) and Raymond and Jiang (1990), is the strength and distri- 
bution of large-scale ascent associated with the PV anomaly. If the ball anomaly is embedded in a vertically sheared flow and is moving relative to air at low levels (e.g., toward the east in a typical midlatitude environment characterized by westerlies increasing with altitude), the low-level air will be forced to ascend along the bulging isentropes as the anomaly approaches. At the same time, this air is also being advected toward the cold side of the vertically sheared flow by the cyclonic winds associated with the PV anomaly, inducing additional isentropic upglide. This can lead to the weakening and eventual removal of CIN, and the subsequent initiation of deep moist convection, through adiabatic cooling if this airstream contains large amounts of water vapor (Jiang and Raymond 1995; Trier et al. 2000). Static inversion incorporates the former process [ascent along bulging isentropes, which Hoskins et al. (1985) called the "vacuum cleaner effect"], but not the effect of advection transverse to the vertically sheared flow. The neglect of the latter effect is only significant if the transversely ascending air parcels have different thermodynamic characteristics than the parcels they are replacing.

The discussion below focuses on changes in CAPE. CIN changes will generally be of the opposite sign and relatively small because CIN is determined by thermodynamic structure close to the lower boundary. From the conventional air parcel perspective, decreases in CIN may be thought of as changes in vertical motion induced by the upper-level anomaly that bring air parcels closer to their level of free convection.

\section{Experiment design}

The presence of background PV gradients $\nabla q_{0}$ (such as that associated with a jet stream or the tropopause) complicates the conceptual model underlying PV diagnosis and distorts the balanced "response" to a PV perturbation discussed in section 2. Invoking an electrostatics analogy, Bishop and Thorpe (1994) and Thorpe and Bishop (1995) demonstrate that the equations relating quasigeostrophic PV (QGPV) and Ertel PV to the balanced flow are isomorphic to the equations relating electrical charge to the potential field, and that an isolated point vortex (expressed in terms of PV) is analogous to a static electrical charge. Their results show that the primary difference between the potential fields induced by linearized $\mathrm{QG}$ and Ertel PV "charges" is that the response (expressed in terms of the balanced perturbation streamfunction, $\left.\Psi^{\prime}\right)$ is modulated in the presence of both vertical and horizontal gradients in background Ertel PV for the latter case and only in the presence of vertical gradients of back- ground QGPV (such as in the vicinity of the tropopause) for the former.

For both the QG and Ertel PV formulations, Bishop and Thorpe (1994) and Thorpe and Bishop (1995), derive solutions for a point charge placed a small distance beneath the tropopause, which is analagous to a dielectric boundary separating two media of differing dielectric properties in electrostatics. Both solutions (see Bishop and Thorpe 1994, their Fig. 4) exhibit notable vertical asymmetry, with the perturbation streamfunction field decaying much more rapidly into the stratosphere than into the troposphere in the QG formulation, and vice versa in the Ertel PV framework. Although Thorpe and Bishop (1995) do not explicitly consider the solution for a point vortex placed near a horizontally oriented PV gradient (such as that associated with a jet stream), the presence of a horizontal background PV gradient would be expected to produce a distortion of the balanced response in the horizontal plane because the solution for an Ertel PV charge is sensitive to polarization in both the horizontal and vertical directions.

The experiments described here are designed to investigate the effect of background horizontal PV gradients and PV anomaly amplitude on the balanced atmospheric response to idealized PV anomalies. Because the immediate concern of this series of papers is the potential effect of PV variations on the development of severe weather, the PV anomalies will be embedded in the analyzed atmospheric state at 1800 UTC 13 March 1990, the afternoon of the Hesston tornado outbreak (Davies et al. 1994) and the subject of Part III. National Centers for Environmental PredictionNational Center for Atmospheric Research (NCEPNCAR) reanalysis gridded data (Kalnay et al. 1996) are interpolated to a $2.5^{\circ} \times 2.5^{\circ}(278 \mathrm{~km} \times 218 \mathrm{~km}) \mathrm{com}-$ putational grid at 50-hPa intervals between 1000 and 50 $\mathrm{hPa}$ for inversion purposes (coarser than in Part I for simplicity). The inversion domain includes all of North America.

The inversion procedure uses the nonlinear balance equations, following Davis and Emanuel (1991). The analyzed atmospheric state is taken to be the basic state, with potential vorticity $q_{0}$, and this $\mathrm{PV}$ is inverted to obtain the balanced height $\left(\Phi_{B}\right)$ and streamfunction $\left(\Psi_{B}\right)$ fields. Idealized PV anomalies $\delta q$ are added to this basic state to obtain the modified PV $q$. Inversion of the PV anomalies produces fields of anomalous balanced height $\left(\delta \Phi_{B}\right)$ and streamfunction $\left(\delta \Psi_{B}\right)$. Further details of the data and inversion procedure are found in Part I (sections 2a-b).

While virtual temperatures are obtainable from the balanced heights, computation of CAPE also requires 
knowledge of the moisture distribution. Here, the moisture distribution is recovered by assuming that mixing ratio is the same function of potential temperature in the original and modified (inverted) soundings (Part I). This assumption is equivalent to assuming that air parcels adjust to a new thermodynamic state associated with a new PV distribution by moving vertically. The CAPE computed from the balanced soundings obtained by inverting the analyzed PV field typically differs by less than $10 \%$ from the actual analyzed CAPE (Part I).

A conceptual framework for understanding how PV modifications affect the 13 March 1990 environment is established by devising several experiments in which simple idealized PV anomalies are constructed and inverted. The simple constructions are created using "grid volume anomalies," each of which is a grid volume $\left(2.5^{\circ} \times 2.5^{\circ} \times 50 \mathrm{hPa}\right)$ in which the $\mathrm{PV}$ is increased or decreased by a specific amount. Because any PV anomaly represented on a grid is ultimately composed of many such volumes, each grid volume PV anomaly can be thought of as a "PV atom," a basic building block from which more complex and realistic PV configurations can be generated. One outcome of the tests of the linearity of the response is an assessment of the degree to which more complex PV distributions may be evaluated as the sum of their PV elements.
Three experiments are performed in which idealized PV anomalies of increasing geometrical complexity are constructed and then added to the observed PV distribution $q_{0}$. The total PV field in each case is simply the sum of $q_{0}$ and the idealized PV anomaly $\delta q$. Because we are considering the effect of tropopause PV anomalies in this study, the PV in each case is perturbed within grid volumes centered vertically about the $300-\mathrm{hPa}$ level. In the first and simplest experiment (EXP1), the $\mathrm{PV}$ is changed within a single grid volume, and the amplitude of this PV anomaly is increased for each successive inversion until linearity breaks down. In the second test (EXP2), the PV is changed at three adjacent grid volumes aligned northwest to southeast (the "PV string" experiment), replicating a highly idealized negatively tilted trough, and the total response due to all three perturbations is compared with that attributable to each separately. The final experiment (EXP3) juxtaposes two PV strings, each of opposite sign, to crudely represent the translation of the negatively tilted PV anomaly constructed in EXP2.

\section{The idealized experiments}

\section{a. Single gridpoint anomaly: EXP1}

The first experiment consists of modifying the observed $\mathrm{PV} q_{0}$ at grid point $\mathrm{A}$ :

$$
\delta q= \begin{cases}(R / 3) q_{0}, & \text { at } \quad\left(x_{0}, y_{0}, p_{k+1}\right),\left(x_{0}, y_{0}, p_{k}\right),\left(x_{0}, y_{0}, p_{k-1}\right) \\ 0, & \text { everywhere else }\end{cases}
$$

where $R$ is an integer multiple representing the PV anomaly strength. The PV perturbation is spread vertically across three contiguous levels so that the anomaly's nondimensional aspect ratio is of order unity. The aspect ratio, $(N / f)(\Delta z / \Delta x)$ (where $N$ is the BruntVäisälä frequency, $f$ is the Coriolos parameter, and $\Delta z$ and $\Delta x$ are measures of the anomaly depth and width, respectively), expresses the ratio of vorticity to stratification in a particular PV anomaly (Wirth 2000; Hoskins et al. 1985). A shallow anomaly (i.e., one whose aspect ratio is small) is manifested primarily as an anomaly in stratification while a tall anomaly is realized mostly through relative vorticity (Wirth 2000). Thus, when the aspect ratio is close to unity, the PV perturbation's response will not be biased to either the vorticity or stratification but rather equally partitioned between the two.

The particular grid volume element perturbed in EXP1 is located on the anticyclonic shear side of the upper-tropospheric jet stream and the resulting pertur- bation streamfunction field $\delta \Psi_{B}$ with $R=1$ potential vorticity unit (PVU; Fig. 2a) is consistent with the location and strength of the single grid volume PV anomaly. The anisotropy of $\delta \Psi_{B}$ is implied by the electrostatics analogy, whereby the perturbation balanced response to a PV anomaly placed on the low-PV side of a transition zone in the background PV is elongated in the direction of the background gradient vector. The $\delta \Phi_{B}$ field is also elliptical in shape, but with its major axis oriented roughly perpendicular to the $\delta \Psi_{B}$ field. The corresponding $\delta$ CAPE distribution, which depends upon $\delta \Phi_{B}$, is also an elliptical patch oriented roughly parallel to the background upper-tropospheric PV gradient.

A spatial diagnosis of the various terms in the balance equation (not shown) reveals that the difference in orientation of the major axes between the $\delta \Psi_{B}$ and $\delta \Phi_{B}$ fields is entirely attributable to the Jacobian term in the nonlinear balance (NLB) equation: 


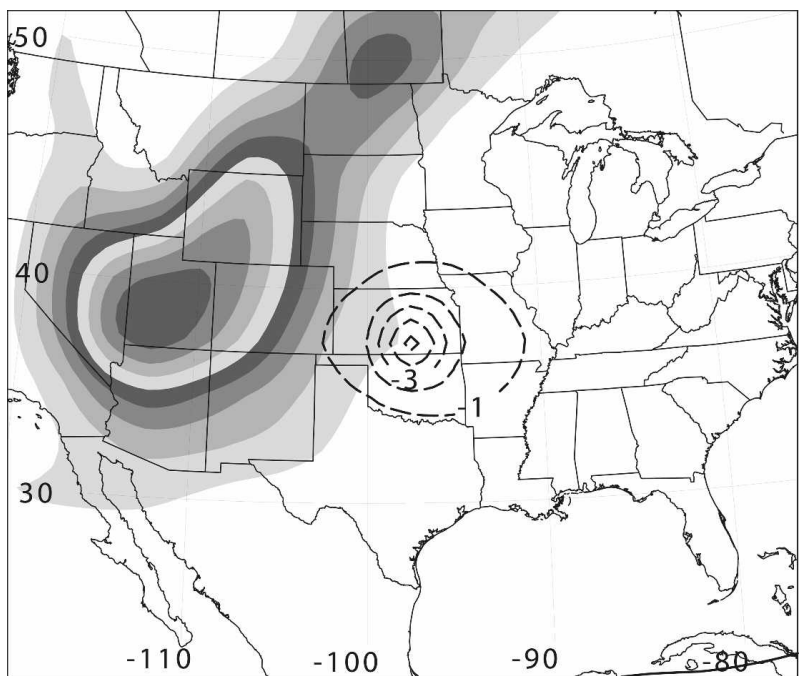

(a) 300 MB EXP 1: PERT PSI, OBS PV

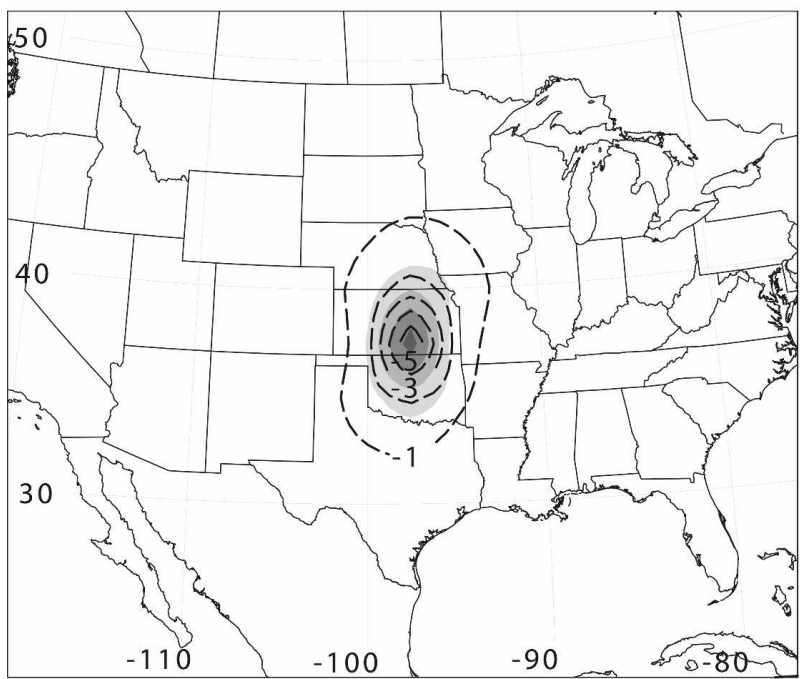

(b) 300 MB EXP 1: PERT Z, CAPE > $8 \mathrm{~J} / \mathrm{KG}$

FIG. 2. (a) Perturbation streamfunction field $\delta \Psi_{B}$ (contoured every $1 \mathrm{~m}^{2} \mathrm{~s}^{-1}$; dashed contours are negative) induced by an idealized single grid volume $\mathrm{PV}$ anomaly twice the original $\mathrm{PV}$ value $(R=1)$, located at $300 \mathrm{hPa}$ and $37.5^{\circ} \mathrm{N}, 97.5^{\circ} \mathrm{W}$ at $1800 \mathrm{UTC} 13$ Mar 1990, shown in relation to the background PV field (shading; only values greater than 1 PVU are filled); (b) the corresponding perturbation geopotential height field (contoured every $1 \mathrm{~m}$ ) and perturbation CAPE distribution (light shaded region, values greater than $8 \mathrm{~J} \mathrm{~kg}^{-1}$ are filled with an increment of $8 \mathrm{~J} \mathrm{~kg}^{-1}$ ).

$$
\frac{2}{a^{4} \cos ^{2} \phi} J_{\lambda, \phi}\left(\frac{\partial \Psi}{\partial \lambda}, \frac{\partial \Psi}{\partial \phi}\right)
$$

Herein lies the primary difference between the QG and NLB systems: in the former, $\delta \Psi_{B}, \delta \Phi_{B}$, and $\delta$ CAPE all would have a radially symmetric horizontal structure. Thus, resorting to a higher order of balance produces a potentially important variation in the spatial structure of the balanced CAPE perturbation. Several additional
PV inversions of single grid volume perturbations placed at other grid points located on the anticyclonic shear side of (and in close proximity to) the uppertropospheric jet were performed (not shown). In every case, the $\delta \Psi_{B}$ field exhibited elongation along the local background PV gradient vector $\nabla q_{0}$ while the major axis of the $\delta \Phi_{B}$ and $\delta$ CAPE fields was roughly perpendicular to $\nabla q_{0}$ as shown at the sample time displayed in Fig. 2.

Similar effects are seen in Parts III and IV when more realistic PV perturbations are applied to the upper troposphere in the vicinity of the jet streams. Alterations to CAPE due to details of the PV structure along the jet lie mainly along the path of the jet and extend farther ahead of the modified PV than to either side.

Multiple PV inversions of the single grid volume PV anomaly were conducted to test whether the CAPE responses to PV perturbations are linear for realistic PV anomaly magnitudes. The results of Thorpe and Bishop (1995) and Birkett and Thorpe (1997) suggest that the inversion of $\mathrm{PV}$ anomalies corresponding to higher-order balance approximations (such as the NLB system) produces a response whose nonlinearity is highly localized and proportional to anomaly strength.

The methodology applied is to change the $300-\mathrm{hPa}$ PV at grid point $\left(\phi_{0}, \lambda_{0}\right)$ by real multiples of the basicstate PV value $q_{0}$ at that point. ${ }^{1}$ For each value of $R$, $\delta \operatorname{CAPE}(R)$ is computed at $\left(\phi_{0}, \lambda_{0}\right)$. Departure from linearity (the error) is quantified with respect to $R=1$ as

$$
\left|\frac{\delta \mathrm{CAPE}(1)-\frac{\delta \mathrm{CAPE}(R)}{R}}{\delta \mathrm{CAPE}(1)}\right| .
$$

If the response of $\delta \mathrm{CAPE}$ is linear with respect to $R$ for the range of values tested, the numerator will equal zero.

Table 1 summarizes the result of inverting the grid volume anomalies of variable strength. The results associated with the inversion of the positive (negative) anomalies are listed in the left (right) half of the table. For positively signed $\delta q$ the response manifested in the $\delta$ CAPE field is highly linear through PV anomalies of strength $R=5$ with departures from linearity of generally less than $1 \%$. However, for negatively signed PV changes, linearity is not as robust. At PV amplitudes of $R=-1$ the departure from linearity is roughly $10 \%$

\footnotetext{
${ }^{1}$ As discussed previously, if the PV is changed by adding an amount $\delta q=R$, that amount is vertically distributed in equal portions of $(R / 3) q_{0}$ at 350,300 , and $250 \mathrm{hPa}$.
} 
TABLE 1. CAPE perturbations and departures from linearity (errors) associated with the inversion of grid box PV anomalies of varying amplitude (measured in PVU).

\begin{tabular}{lcccccc}
\hline \hline \multicolumn{3}{c}{$\delta q>0$} & & \multicolumn{3}{c}{$\delta q<0$} \\
\cline { 1 - 3 } \cline { 5 - 7 }$R$ & $\delta$ CAPE & Error (\%) & & $R$ & $\delta$ CAPE & Error (\%) \\
\cline { 1 - 3 } 1 & 35.2 & - & & -0.4 & -14.7 & - \\
2 & 70.7 & 0 & & -0.6 & -21.2 & 4 \\
3 & 106.7 & 1 & & -0.8 & -26.9 & 9 \\
4 & 141.9 & 1 & & -1.0 & -32.9 & 10 \\
5 & 177.5 & 1 & & & \\
\hline
\end{tabular}

and this error gradually increases as negative PV perturbation magnitude is increased (not shown).

It should be noted that Juckes and Smith (2000) find that the CAPE response is a nonlinear function of tropopause temperature amplitude, in contrast to the quasi-linear response demonstrated above. The most likely reason for the discrepancy is the difference in how the PV anomalies are specified. When PV anomalies are specified as changes in the tropopause temperature, the altitude of the center of the PV anomaly becomes progressively lower (higher) as the anomaly becomes more cyclonic (anticyclonic). Temperatures respond to both the strength and proximity of the PV anomaly. In the idealized inversions described here, the dynamic tropopause will descend as the amplitude of the anomaly increases, but the centroid of the anomaly will remain at the same level. Both approaches are valid, and depend partly upon whether one prefers to measure the strength of a PV anomaly by its PV perturbation or by its tropopause potential temperature perturbation. As the anomaly attains very large size and amplitude, the Juckes and Smith (2000) formulation is likely to be more realistic, leading to a nonlinear increase in the convective impact of the anomaly.

\section{b. String $P V$ anomaly: EXP2}

The second experiment probing the linearity of various artificial PV constructions was chosen so as to correspond to the effects of adding a "negatively tilted" PV anomaly to the flow. Many significant severe convective episodes are associated with upper-level mobile troughs exhibiting an axis with a pronounced upshear tilt (Macdonald 1976). The experiment is conducted by changing the PV at three adjacent grid points aligned northwest to southeast. The locations at which the PV is perturbed are chosen so that the anomaly protrudes southward and eastward into Texas, a region possessing nonnegligible CAPE (Fig. 3a). At each of the three perturbed locations, the PV is increased by $3 \mathrm{PVU}$, a magnitude chosen to ensure a large CAPE response.

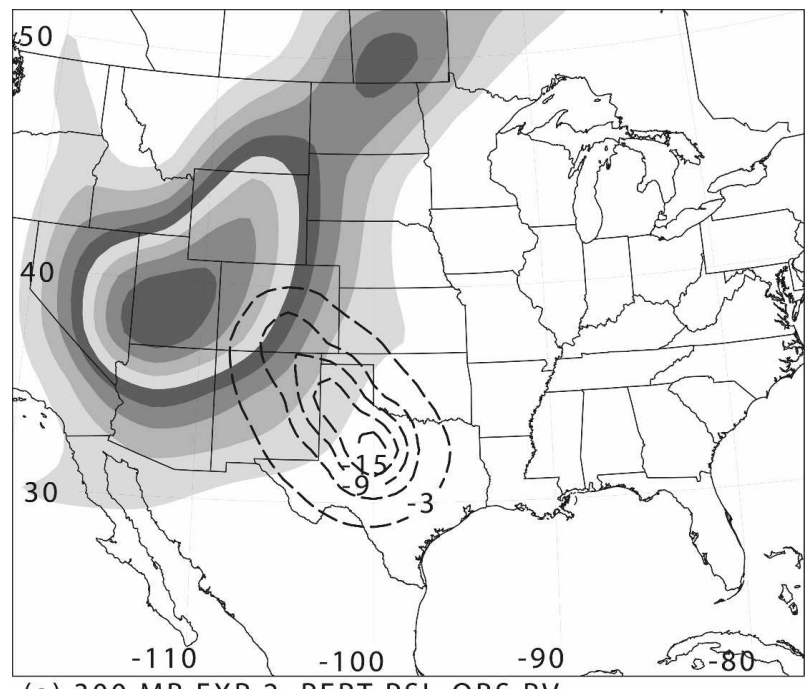

(a) 300 MB EXP 2: PERT PSI, OBS PV

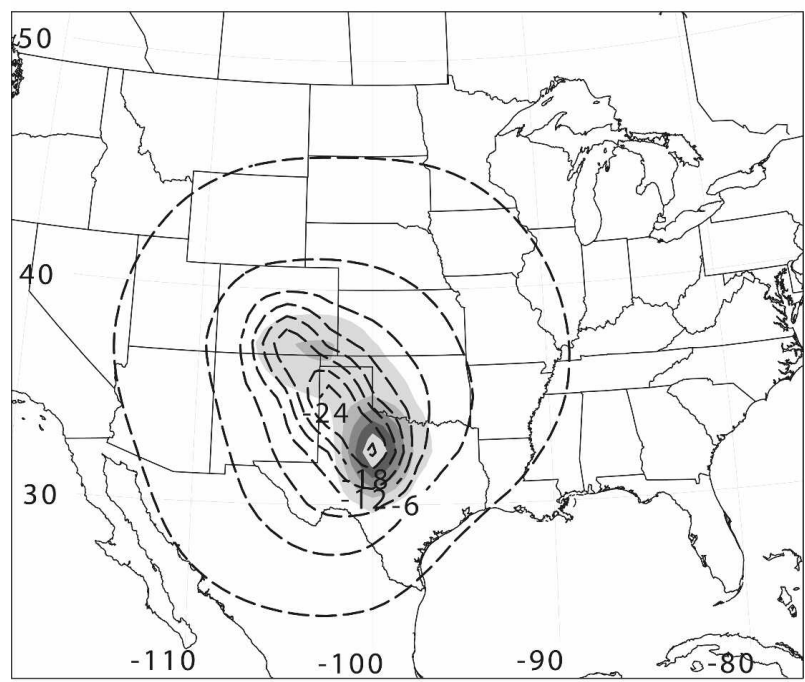

(b) $300 \mathrm{MB}$ EXP 2: PERT Z, CAPE > $25 \mathrm{~J} / \mathrm{KG}$

FIG. 3. (a) As in Fig. 2, but for a negatively tilted string PV anomaly composed of three PV anomalies (of amplitude 3 PVU each) aligned from southern $\mathrm{CO}$ to central TX. (b) The perturbation CAPE field in is filled for values exceeding $+25 \mathrm{~J} \mathrm{~kg}^{-1}$ and the fill interval is every $25 \mathrm{~J} \mathrm{~kg}^{-1}$.

As expected, the $\Phi_{B}$ and $\Psi_{B}$ perturbations indeed exhibit a pronounced negative tilt, with $\delta \Phi_{B}$ showing slightly more elongation across the background PV gradient vector than $\delta \Psi_{B}$ (Fig. 3b), consistent with EXP1. Compare, for example, the shapes across Oklahoma, Texas, and northern Mexico of the $\delta \Psi_{B}=3 \mathrm{~m}^{2} \mathrm{~s}^{-1}$ isopleth in Fig. 3a and the $\delta \Phi_{B}=6 \mathrm{~m}$ isopleth in Fig. 3b.

Linearity with respect to CAPE is once again examined by comparing the $\delta \mathrm{CAPE}$ associated with the total perturbation PV field (i.e., all three gridpoint anomalies together) to that obtained from inverting each of the three anomalies individually and summing the 
CAPE perturbations. The results, displayed in Fig. 4a, again demonstrate that linearity is closely preserved in the $\delta$ CAPE field because the sum of the individual inversions (light contours) yields $\delta \mathrm{CAPE}$ that is nearly identical to that computed from the inversion of the sum of the grid volume PV anomalies composing the total perturbation (dark contours). Two additional subsidiary experiments were performed with the "string anomaly" doubled (6 PVU per grid volume) and tripled (9 PVU per grid volume) in magnitude, respectively. Once again, linearity was found to be very closely preserved in both cases (not shown).

The spatial distribution of $\delta$ CAPE shown in Fig. $3 b$ is asymmetrical, with much higher values centered over northwestern Texas and negligible values farther northwest, despite the fairly uniform values of $\delta \Phi_{B}$ associated with the idealized forcing line. The asymmetry in perturbation CAPE is produced by horizontal variations in the background PV along the forcing line, which is oriented (by construction) nearly parallel to the local basic-state PV gradient. The southeasternmost point vortex, located near Abilene, Texas (refer to Fig. $3 \mathrm{a}$ ), is located in the troposphere (well to the southeast of the 1.5-PVU contour at $300 \mathrm{hPa}$ ) while the northwesternmost point vortex is located within the stratospheric air just southeast of the highest PV values within the large-scale upper-level trough centered over Utah. As a consequence, the southeasternmost point vortex represents a much greater fractional increase in PV than the ones closer to the center of the stratospheric extrusion, resulting in a much stronger balanced response to the southeast. Moreover, perturbing the stratospheric PV distribution cannot strongly affect the CAPE within the underlying columns because the cooling associated with stratospheric PV anomalies is confined to relatively high altitudes of the troposphere. Forecasters should avoid being drawn to PV variations within the lower stratosphere and instead pay close attention to those PV anomalies embedded within or adjacent to a low-PV environment in the upper troposphere, because the latter anomalies will have much greater impact on the tropospheric winds, temperature, and stability.

\section{c. Translational PV anomaly: EXP3}

A final experiment was designed to bridge the gap between the simple idealized PV constructions of EXP1 and EXP2, created to represent simple changes to the observed upper-tropospheric PV distribution, and realistic PV modifications. There exist several canonical kinematic properties by which a patch of fluid can be transformed: translation, rotation, expansion, deformation, or a combination of any or all of these. For EXP3,

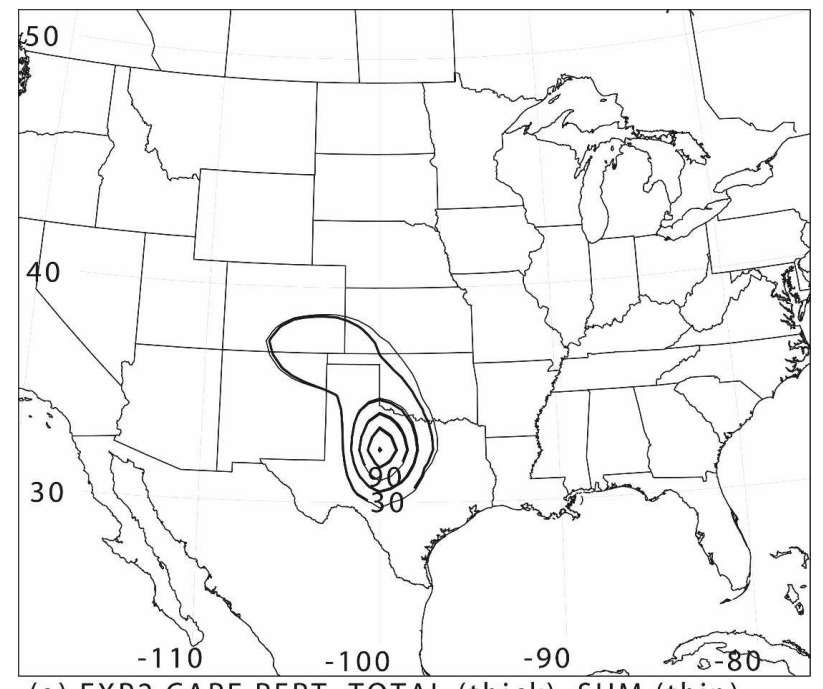

(a) EXP2 CAPE PERT: TOTAL (thick), SUM (thin)

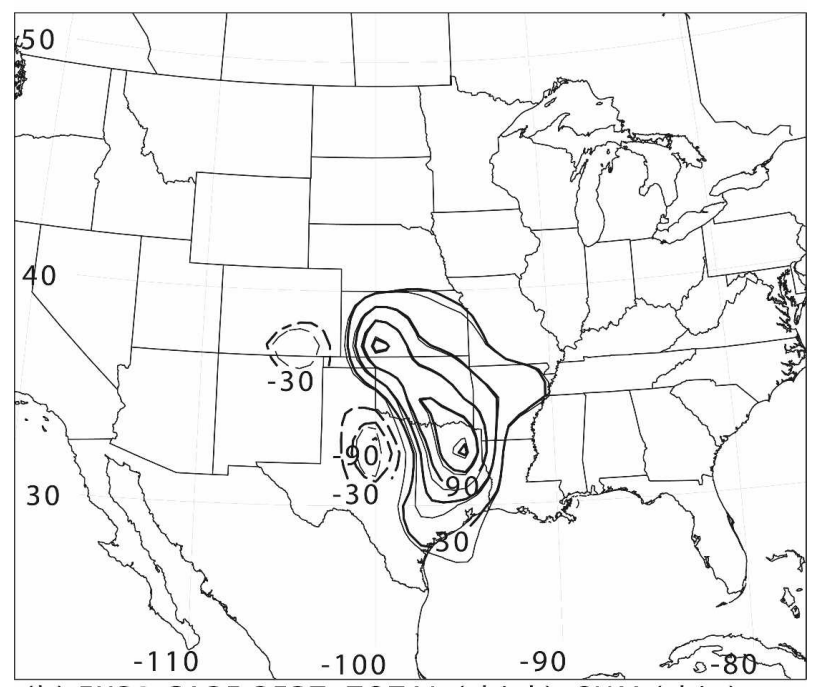

(b) EXP3 CAPE PERT: TOTAL (thick), SUM (thin)

FIG. 4. CAPE perturbations associated with (a) the sum of the separate inversions of each PV anomaly (thin contours) and the inversion of the sum of the three PV anomalies (thick contours) in EXP2; (b) as in (a), but for EXP3 (negative values dashed).

the simplest of these transformations, translation, is chosen to represent the consequences of an error in position of an upper-level trough. The dipole anomaly is achieved by first adding to $q_{0}$ a positive monopole string (as was done in EXP2) and the resulting PV distribution then serves as the new background PV field. The PV anomaly consists of a positive monopole string placed two grid points ( $5^{\circ}$ of longitude) east of the original string, and a negative monopole string anomaly at the location of the original string. This positivenegative dipole string anomaly represents a two grid point "error" in the location of the original string. When inverted, a dipolar streamfunction perturbation 
is produced (Fig. 5a) with negative (positive) perturbations at the locations of the positive (negative) PV strings. The corresponding geopotential and CAPE perturbations (Fig. 5b) are somewhat elongated across the background PV gradient vector (as in EXP1 and EXP2) with the strongest CAPE anomaly once again produced in the tropospheric air southeast of the PV gradient, where the largest fractional PV increase occurs.

Two additional piecewise inversions are performed in EXP3: one of the negatively signed PV string $\left(\delta q_{-}\right)$ and one of the positively signed string $\left(\delta q_{+}\right)$. Linearity is examined by computing and comparing the CAPE perturbations attributable to the sum of the latter two inversions with that of the inversion of the total dipole anomaly. The total and sum CAPE changes, displayed in Fig. 4b, support the hypothesis that simple translation indeed produces a linear response in the CAPE field at these magnitudes, because the total CAPE anomaly (light contours) is nearly identical to the sum of CAPE perturbations associated with the negative and positive string anomalies (dark contours). Not surprisingly, the primary effect of moving the uppertropospheric "string" PV anomaly downstream is to induce an increase in the balanced CAPE over the central plains in conjunction with the associated decrease in upper-tropospheric $\Phi_{B}$ there. As in EXP2, the largest CAPE increase is realized at the southeasternmost portion of the eastward-shifted positive string anomaly (Fig. 5) and a slight eastward extension of the positive CAPE anomaly away from the trough axis is evident (Fig. 5b).

The foregoing experiments demonstrate that it is possible to understand changes in the CAPE field in a systematic manner given changes to the uppertropospheric PV distribution. Moreover, it can be assumed that $\delta q$ obtained by more complicated manipulations of $q_{0}$, such as rotation and/or deformation of the PV field, will also yield a quasi-linear response in CAPE within the NLB PV inversion framework. This conceptual framework will be applied in the companion papers (Parts III and IV), in which individual case studies are analyzed. As stated earlier, because of the inherently nonlinear dependence of the shear parameters on the winds, the effect of changing the uppertropospheric PV on the vertical shear parameters computed from the balanced wind will be assessed in the context of the case studies.

\section{Summary}

A conceptualization based on the cold-core upperlevel cyclone by Thorpe (1986) asserts that intensifying

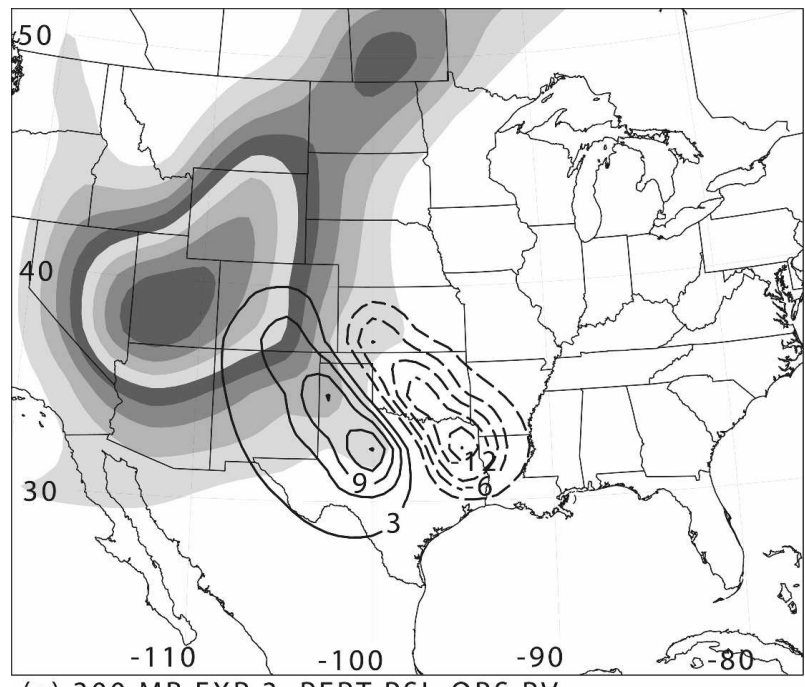

(a) 300 MB EXP 3: PERT PSI, OBS PV

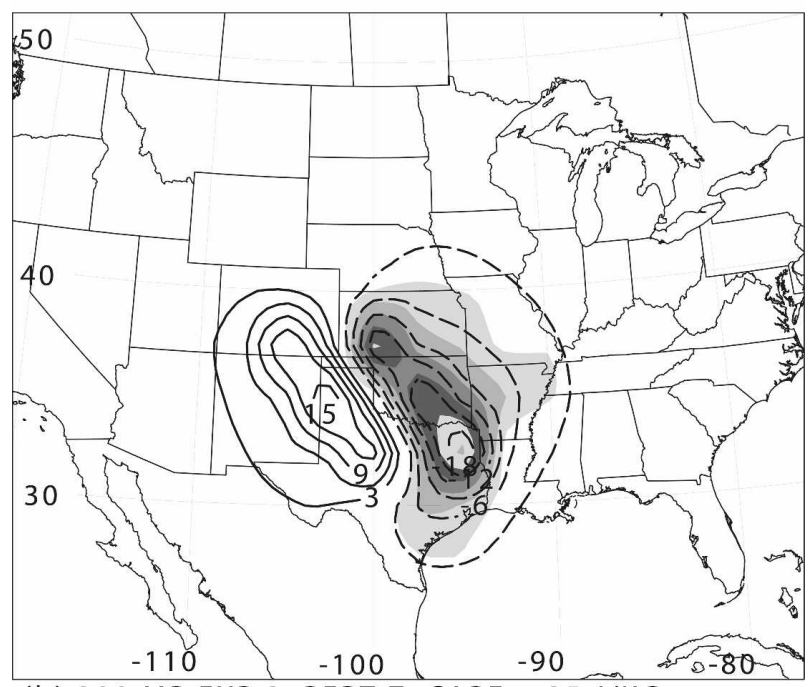

(b) 300 MB EXP 3: PERT Z, CAPE > $25 \mathrm{~J} / \mathrm{KG}$

FIG. 5. As in Fig. 3, but for two opposite signed string PV anomalies representing the effects of moving an idealized negatively tilted trough eastward. The strings are composed of three PV anomalies each, along northwest-southeast oriented lines, with negative PV anomalies to the west. (a) The corresponding perturbation streamfield function (contours, negative values are dashed). (b) The geopotential (negative values are dashed; interval is $3 \mathrm{~m}$ ) and CAPE anomalies (values exceeding $+25 \mathrm{~J} \mathrm{~kg}^{-1}$ and filled every $25 \mathrm{~J} \mathrm{~kg}^{-1}$ ) are shown.

(weakening) a positive upper-level PV anomaly produces a concomitant decrease (increase) in stratification beneath and adjacent to the anomaly, as well as increased (decreased) vertical shear on the flanks of the anomaly. The heuristic generalizations drawn from this simple conceptual model carry over to a realistic environment, as demonstrated in a series of idealized experiments in which geometrically simple PV anomalies are constructed and inverted to obtain the balanced 
CAPE perturbations attributable to each anomaly. Despite numerous nonlinearities inherent in the definition of CAPE and in the balance constraint relating the PV to the balanced flow in the NLB framework, the experiments demonstrate that, in fact, the CAPE responds to PV perturbations in a quasi-linear fashion.

While it is well known that a more intense trough should lead to stronger winds, cooler tropospheric temperatures, and stronger vertical motion, the PV-based approach taken here identifies asymmetries and other details that might otherwise not be apparent. Regardless of the order of balance chosen, the presence of background PV gradients modulates the balanced flow solutions associated with PV perturbations. The electrostatics isomorphisms developed by Bishop and Thorpe (1994) and Thorpe and Bishop (1995) anticipate how the $\delta \Psi_{B}$ field is spatially distorted in the vicinity of a PV gradient, such as that associated with the tropopause or a jet stream. It is found that the perturbation streamfunction field attributable to an isolated Ertel's PV "point charge" placed near a jet stream is an ellipse whose major axis is parallel to the local background $\mathrm{PV}$ gradient vector $\nabla q_{0}$. The nonlinear relationship between $\Psi$ and $\Phi$ inherent in the NLB system distorts the $\delta \Phi_{B}$ field perpendicular to $\nabla q_{0}$. Thus, perturbing the PV near a jet stream induces far-field CAPE changes (which depend on $\delta \Phi_{B}$ ) that extend farther along the jet than across it, and far-field shear changes (which depend on $\delta \Psi_{B}$ ) that extend farther across the jet than along it.

Three simple idealized experiments are conducted to determine the nature of CAPE responses to uppertropospheric PV changes. The experiments demonstrate that, in practice, the CAPE does vary quasilinearly with PV anomaly amplitude, even for relatively complex anomaly shapes possessing moderate disturbance amplitudes. It is also clear from the experiments that the response to anomalies embedded in the upper troposphere, on the anticyclonic shear side of the jet, is much larger than the response to anomalies embedded in the lower stratosphere, on the cyclonic shear side of the jet. This is to be expected, because the comparatively large stratification in the lower stratosphere makes the penetration depth much smaller than in the upper troposphere, where both inertial and static stability are low.

These results suggest that "PV thinking" (Hoskins et al. 1985) may be applied directly to possible analysis or model errors in the upper-tropospheric PV field without much concern for possible nonlinear effects in the far-field response. A somewhat suppressed response was only found when the local PV field was substantially reduced from its original value, implying that the effect of strongly negative small-scale PV perturbations is not as great as simple linear thinking would suggest. Effects on the thermodynamic fields may be expected to be concentrated primarily along the jet axis, while effects on the kinematic fields may be expected to be aligned normal to the jet axis. These departures from symmetry, while interesting dynamically, may not have much operational relevance apart from the general rule that wind perturbations will extend farther from the path of a PV anomaly embedded in a jet than will temperature perturbations.

Having developed a conceptual understanding of how PV perturbations will affect the severe convective environment, the next step is to apply PV inversion and convective index diagnosis to actual severe weather outbreaks. In Part III, the techniques described in Part I are applied to the Hesston severe weather outbreak and the influence of the intensity of the upper-level mobile trough on instability, shear, and associated severe weather is examined in detail. In Part IV, a second event is examined, permitting verification of the basic principles gleaned from Part III as well as allowing comparison with a mesoscale modeling study that investigated similar issues.

Acknowledgments. The authors thank Christopher Davis for providing the software used in inverting the $\mathrm{PV}$, as well as for providing invaluable assistance with the use of the prognostic balance equation solver. This research was supported by the National Science Foundation through Grant ATM-0089906.

\section{REFERENCES}

Birkett, H. R., and A. J. Thorpe, 1997: Superposing semi-geostrophic potential vorticity anomalies. Quart. J. Roy. Meteor. Soc., 123, 2157-2163.

Bishop, C. H., and A. J. Thorpe, 1994: Potential vorticity and the electrostatic analogy: Quasi-geostrophic theory. Quart. J. Roy. Meteor. Soc., 120, 713-731.

Davies, J. M., C. A. Doswell, D. F. Burgess, and J. F. Weaver, 1994: Some noteworthy aspects of the Hesston, Kansas, tornado family of 13 March 1990. Bull. Amer. Meteor. Soc., 75, 1007-1017.

Davis, C. A., and K. A. Emanuel, 1991: Potential vorticity diagnostics of cyclogenesis. Mon. Wea. Rev., 119, 1929-1953.

_, E. D. Grell, and M. A. Shapiro, 1996: The balanced dynamical nature of a rapidly intensifying oceanic cyclone. Mon. Wea. Rev., 124, 3-26.

Gold, D. A., and J. W. Nielsen-Gammon, 2008a: Potential vorticity diagnosis of the severe convective regime. Part I: Methodology. Mon. Wea. Rev., 136, 1565-1581.

$\longrightarrow$, and - , 2008b: Potential vorticity diagnosis of the severe convective regime. Part III: The Hesston tornado outbreak. Mon. Wea. Rev., 136, 1593-1611.

$\longrightarrow$, and $-2008 \mathrm{c}$ : Potential vorticity diagnosis of the severe convective regime. Part IV: Comparison with modeling simu- 
lations of the Moore tornado outbreak. Mon. Wea. Rev., 136, $1612-1629$.

Griffiths, M., A. J. Thorpe, and K. A. Browning, 2000: Convective destabilization by a tropopause fold diagnosed using potential-vorticity inversion. Quart. J. Roy. Meteor. Soc., 126, 125144.

Hoskins, B. J., M. E. McIntyre, and A. W. Robertson, 1985: On the use and significance of isentropic potential vorticity maps. Quart. J. Roy. Meteor. Soc., 111, 877-946.

Jiang, H., and D. J. Raymond, 1995: Simulation of a mature mesoscale convective system using a nonlinear balance model. $J$. Atmos. Sci., 52, 161-175.

Juckes, M., 1999: The structure of idealized upper-tropospheric shear lines. J. Atmos. Sci., 56, 2830-2845.

— , and R. K. Smith, 2000: Convective destabilization by upperlevel troughs. Quart. J. Roy. Meteor. Soc., 126, 111-123.

Kalnay, E., and Coauthors, 1996: The NCEP/NCAR 40-Year Reanalysis Project. Bull. Amer. Meteor. Soc., 77, 437-471.

Klemp, J. B., 1987: Dynamics of tornadic thunderstorms. Annu. Rev. Fluid Mech., 19, 369-402.

Macdonald, N. J., 1976: On the apparent relationship between convective activity and the shape of $500 \mathrm{mb}$ troughs. Mon Wea. Rev., 104, 1618-1622.

Morgan, M. C., and J. W. Nielsen-Gammon, 1998: Using tropo- pause maps to diagnose midlatitude weather systems. Mon. Wea. Rev., 126, 2555-2579.

Raymond, D. J., and H. Jiang, 1990: A theory for long-lived mesoscale convective systems. J. Atmos. Sci., 47, 3067-3077.

Roebber, P. J., D. M. Schultz, and R. Romero, 2002: Synoptic regulation of the 3 May 1999 tornado outbreak. Wea. Forecasting, 17, 399-429.

Shapiro, M. A., and D. Keyser, 1990: Fronts, jet streams, and the tropopause. Extratropical Cyclones: The Erik Palmen Memorial Volume, C. W. Newton and E. O. Holopainen, Eds., Amer. Meteor. Soc., 167-191.

Thorpe, A. J., 1986: Synoptic-scale disturbances with circular symmetry. Mon. Wea. Rev., 114, 1384-1389.

— static analogy: Ertel-Rossby formulation. Quart. J. Roy. Meteor. Soc., 121, 1477-1495.

Trier, S. B., C. A. Davis, and W. C. Skamarock, 2000: Long-lived mesoconvective vortices and their environment. Part II: Induced thermodynamic destabilization in idealized simulations. Mon. Wea. Rev., 128, 3396-3412.

Wirth, V., 2000: Thermal versus dynamical tropopause in upper tropospheric balanced flow anomalies. Quart. J. Roy. Meteor. Soc., 126, 299-317. 